\title{
Aumento de la mortalidad por cáncer de colon en Chile, 1990-2003
}

\author{
Andrés Donoso $D^{a}$, Luis Villarroel del $\mathbf{P}^{1, b}$, G eorge Pinedo $M^{2}$. \\ Increase in colon cancer mortality \\ rates in Chile, during the period \\ 1990-2003
}

Background: In Chile, colorectal cancer is the third cause of mortality due to digestive cancer. Aim: To assess the evolution of colon cancer mortality rates in the period 1990-2003. Material and methods: Information was obtained from demography and vital statistics yearbooks, published by the Chilean National Statistics Institute. Mortality tendecies and slopes, were calculated using Pearson correlation analysis and linear regression. Proportions were compared using Chi square. Results: There was a significantly rising tendency in mortality for colon cancer in the general population ( $r=0.964, p<0.001)$, in men $(r=0.926, p<0.001)$ and in women $(\mathrm{r}=0.943, \mathrm{p}<0.001)$. This tendency was not modified if rates were corrected by age. Mortality among women was significantly higher in all study years. The increase in mortality rates in the period was $0.175 / 100,000$ inhabitants per year. The higher mortality rates were observed during 2003, reaching 6.2/100,000 inhabitants. Conclusions: There was a steady increase in colon cancer mortality during the studied period (Rev Méd Chile 2006; 134: 152-8).

(Key words: Colonic neoplasms; Mortality; Rectal neoplasms)

Recibido el 26 de enero, 2005. Aceptado el 16 de agosto, 2005.

Departamentos de ${ }^{1}$ Salud Pública y de ${ }^{2}$ Cirugía Digestiva, Facultad de Medicina, Pontificia Universidad Católica de Chile.

anterno Facultad de Medicina, Pontificia Universidad Católica de Chile.

bEstadístico, Pontificia Universidad Católica de Chile.

E cáncer es la segunda causa de muerte en Chile y el digestivo es responsable de $46,2 \%$ del total de los fallecimientos por esa causa; de ellos, el cáncer colorrectal es la tercera causa de muerte después del gástrico y biliar ${ }^{1}$.

En el mundo, cada año se registran aproximadamente 1 millón de nuevos casos de cáncer colorrectal y medio millón de muertes, con una tasa cruda mundial de mortalidad de 8,1/100.000 habitantes; afecta principalmente a las regiones más desarmolladas (25,1/100.000 habitantes), mientras que en las de menor desarrollo la tasa es significativamente infe-

Correspondencia a: Andrés Donoso Durante. Huara-Huara 1942, Las Condes. E-mail: aidonoso@puc.cl nior (3,9/100.000 habitantes) $)^{2}$. Sin embargo, países desarrollados como Estados Unidos de Norteamérica (EE.UU) $^{3}$, Canadá 4 y algunos de la Comunidad Europea ${ }^{5}$ presentan en la actualidad incidencias y tasas de mortalidad por cáncer colorrectal en descenso, lo que sería atribuible al diagnóstico pre$\operatorname{coz}^{6,7}$, al mejor tratamiento y a los cambios de los hábitos nutricionales de la población ${ }^{8}$.

En Chile no hay estudios de incidencia y mortalidad por cáncer colorrectal, como tampoco hay programas de salud pública para su prevención y detección precoz, de ahí que los estudios orientados a establecer un diagnóstico poblacional de incidencia o de mortalidad serían útiles para el desarrollo de políticas de salud destinadas al control de esta enfermedad. 
El objetivo de este estudio es evaluar la tendencia de la mortalidad por cáncer de colon en Chile en el período 1990-2003.

\section{MATERIAL Y MÉTODO}

Se estudió la tendencia de la tasa de mortalidad por cáncer de colon en Chile en el período 1990-2003. Las tasas de mortalidad general, por sexo y rangos de edad, se calcularon en relación a la población estimada al 30 de junio de cada año, utilizando los datos crudos obtenidos de los Anuarios de Estadísticas Vitales correspondientes al período estudiado y publicados por el Instituto Nacional de Estadísticas de Chile ${ }^{9}$. Las tasas de mortalidad del período fueron ajustadas por edad para controlar el efecto del envejecimiento poblacional, utilizando como población estándar la correspondiente a 2003. Desde 1997, Chile adoptó la Décima Clasificación Internacional de Enfermedades y Causas de Defunción ${ }^{10}$, este cambio no afectó el estudio, ya que en lo referente a cáncer de colon sólo hubo cambio de la codificación y no del contenido. Se excluyeron del análisis a las muertes por cáncer rectal, por evidencia que señala que estos cánceres no comparten la totalidad de los factores de riesgos y los compartidos tienen diferente poder estadístico en su asociación ${ }^{11}$.

El estudio de tendencia y de pendiente del período se efectuó mediante análisis de comelación de Pearson y de regresión lineal, respectivamente. La comparación de proponciones mediante Chi cuadrado $\left(\chi^{2}\right)$ con corrección de Yates. Se estableció un nivel de significación estadística de $\mathrm{p} \varangle 0,05$.

\section{RESULTADOS}

En el período 1990-2003, hubo 9.968 muertes por cáncer de colon; 4.096 (41,1\%) fallecimientos correspondieron a hombres y $5.872(58,9 \%)$ a mujeres.

Las tasas crudas de mortalidad por cáncer de colon en la población general y por sexo se presentan en la Tabla 1. Hubo un número mayor de muertes de mujeres que de hombres en cada uno de los años del período estudiado, diferencia estadísticamente significativa. Las menores y mayores tasas de mortalidad se registraron en los años extremos del período.

Tabla 1. N úmero de muertes y tasas crudas de mortalidad (x 100.000) por cáncer de colon en la población general y por sexo de los fallecidos. Chile 1990-2003

\begin{tabular}{|llcc|}
\hline Año & $\begin{array}{c}\text { General } \\
\mathrm{n}(\text { tasa })\end{array}$ & $\begin{array}{c}\text { Hombres } \\
\mathrm{n}(\text { tasa })\end{array}$ & $\begin{array}{c}\text { Mujeres* } \\
\mathrm{n}(\text { tasa })\end{array}$ \\
\hline 1990 & $489(3,7)$ & $186(2,9)$ & $303(4,5)$ \\
1991 & $530(4,0)$ & $221(3,3)$ & $309(4,6)$ \\
1992 & $545(4,0)$ & $221(3,3)$ & $324(4,7)$ \\
1993 & $579(4,2)$ & $255(3,7)$ & $324(4,6)$ \\
1994 & $631(4,5)$ & $258(3,7)$ & $373(5,3)$ \\
1995 & $658(4,6)$ & $268(3,8)$ & $390(5,4)$ \\
1996 & $697(4,8)$ & $261(3,7)$ & $436(6,0)$ \\
1997 & $768(5,3)$ & $324(4,5)$ & $444(6,0)$ \\
1998 & $697(4,7)$ & $273(3,7)$ & $424(5,7)$ \\
1999 & $799(5,3)$ & $318(4,3)$ & $481(6,3)$ \\
2000 & $863(5,7)$ & $335(4,5)$ & $528(6,9)$ \\
2001 & $876(5,7)$ & $395(5,2)$ & $481(6,2)$ \\
2002 & $860(5,5)$ & $359(4,7)$ & $501(6,4)$ \\
& $976(6,2)$ & $422(5,4)$ & $554(7,0)$ \\
\hline
\end{tabular}

Fuente: Instituto Nacional de Estadísticas ${ }^{9}$.

*Chi cuadrado: $\mathrm{p}<0,05$ para cada año del período. 
Las tasas de mortalidad crudas y ajustadas por edad, en la población general y por sexo, se presentan respectivamente en las Figuras 1, 2 y 3. La tasa cruda de mortalidad general por cáncer de colon presentó una tendencia ascendente y significativa ( $r=0,964 ; p<0,001)$, similar tendencia se observó en la mortalidad de mujeres ( $\mathrm{r}=0,943$; $\mathrm{p}$ $<0,001)$ y hombres ( $r=0,926$; $p<0,001)$. Al ajustar las tasas por edad se observa que persiste una tendencia ascendente y significativa, tanto en la población general $(r=0,885 ; p<0,001)$, como en hombres $(\mathrm{r}=0,823$; $\mathrm{p}<0,001)$ y mujeres $(\mathrm{r}=0,800$; $\mathrm{p}$ $<0,001)$.
La pendiente del período para las tasas crudas y ajustadas por edad es ascendente y estadísticamente significativa. La primera muestra un incremento anual de la tasa de mortalidad de 0,175/ 100.000 habitantes y la segunda de 0,098/100.000 habitantes (Tabla 2).

En la Figura 4 se presenta la mortalidad por sexo según rangos de edad de los fallecidos en 1990 y 2003. Se aprecia en ambos sexos un aumento sostenido de la tasa de mortalidad desde los 50 años, correspondiendo a 91,4\% de los fallecidos en 1990 y $92,8 \%$ en 2003, hasta alcanzar en los mayores de 79 años la mortalidad más alta.

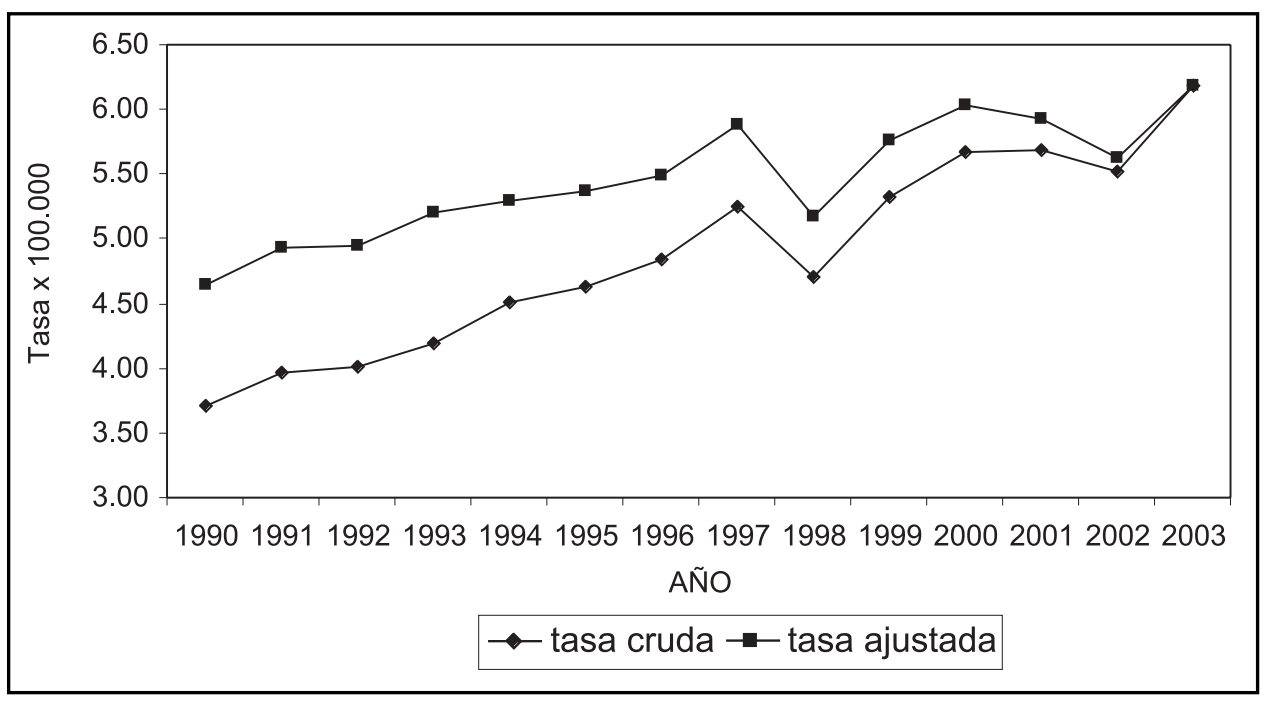

FIgURA 1. Tasas de mortalidad general por cáncer de colon, crudas y ajustadas por edad. Chile 1990-2003.

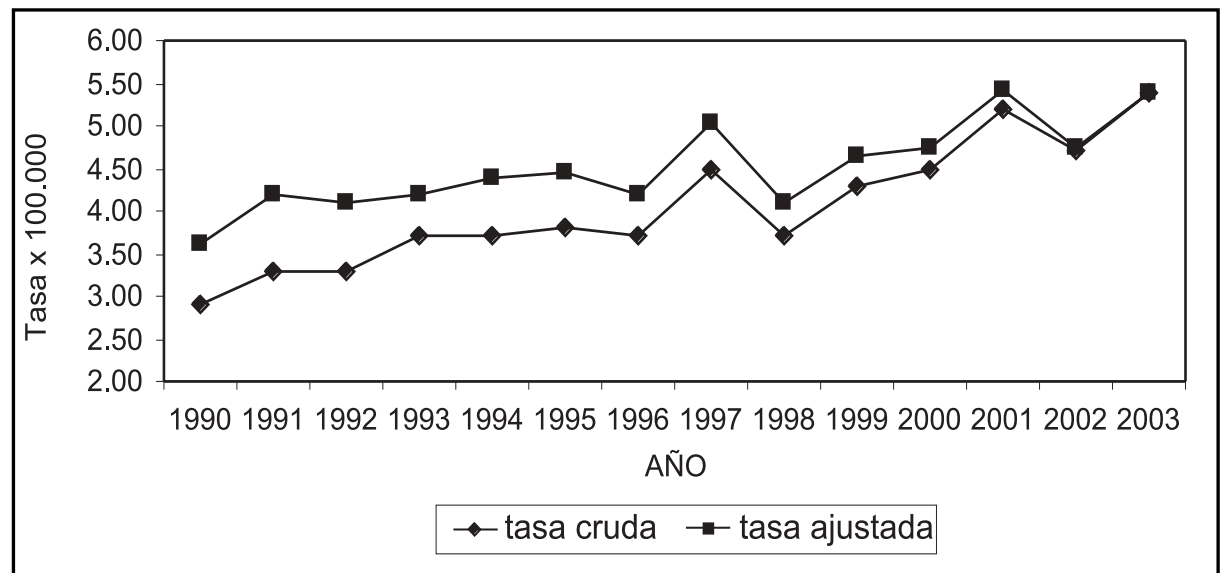

FiguRA 2. Tasas de mortalidad por cáncer de colon en hombres, crudas y ajustadas por edad. Chile, 1990-2003. 


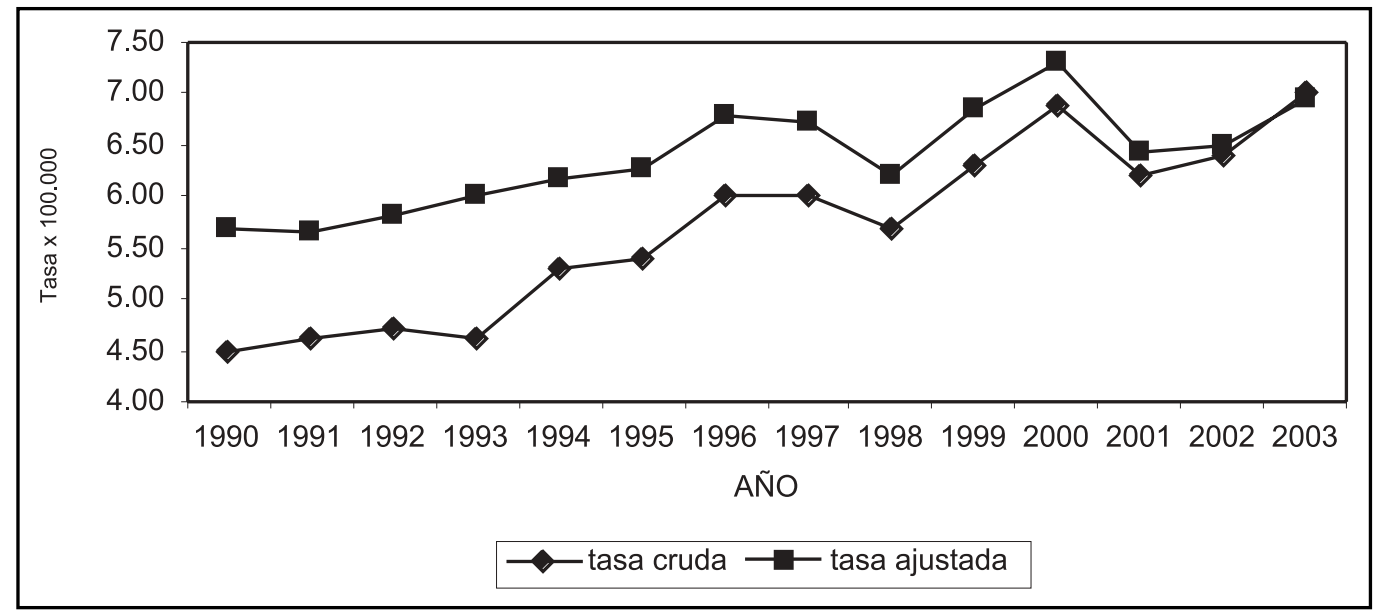

Figura 3. Tasas de mortalidad por cáncer de colon en mujeres, crudas y ajustadas por edad. Chile, 1990-2003.

Tabla 2. Análisis de pendiente de la tendencia de la mortalidad por cáncer de colon para el período 1990-2003.

\begin{tabular}{|lccc|}
\hline & $\begin{array}{c}\text { Tasas crudas } \\
\text { (pendiente } \pm \mathrm{EE} \text { ) }\end{array}$ & $\begin{array}{c}\text { Tasas ajustadas } \\
\text { (pendiente } \pm \mathrm{EE} \text { ) }\end{array}$ & Valor $\mathrm{p}$ \\
\hline Población general & $0,175 \pm 0,01$ & $0,098 \pm 0,01$ & $<0,001$ \\
Hombres & $0,163 \pm 0,01$ & $0,102 \pm 0,02$ & $<0,001$ \\
Mujeres & $0,193 \pm 0,02$ & $0,096 \pm 0,02$ & $<0,001$ \\
\hline
\end{tabular}

EE: error estándar.

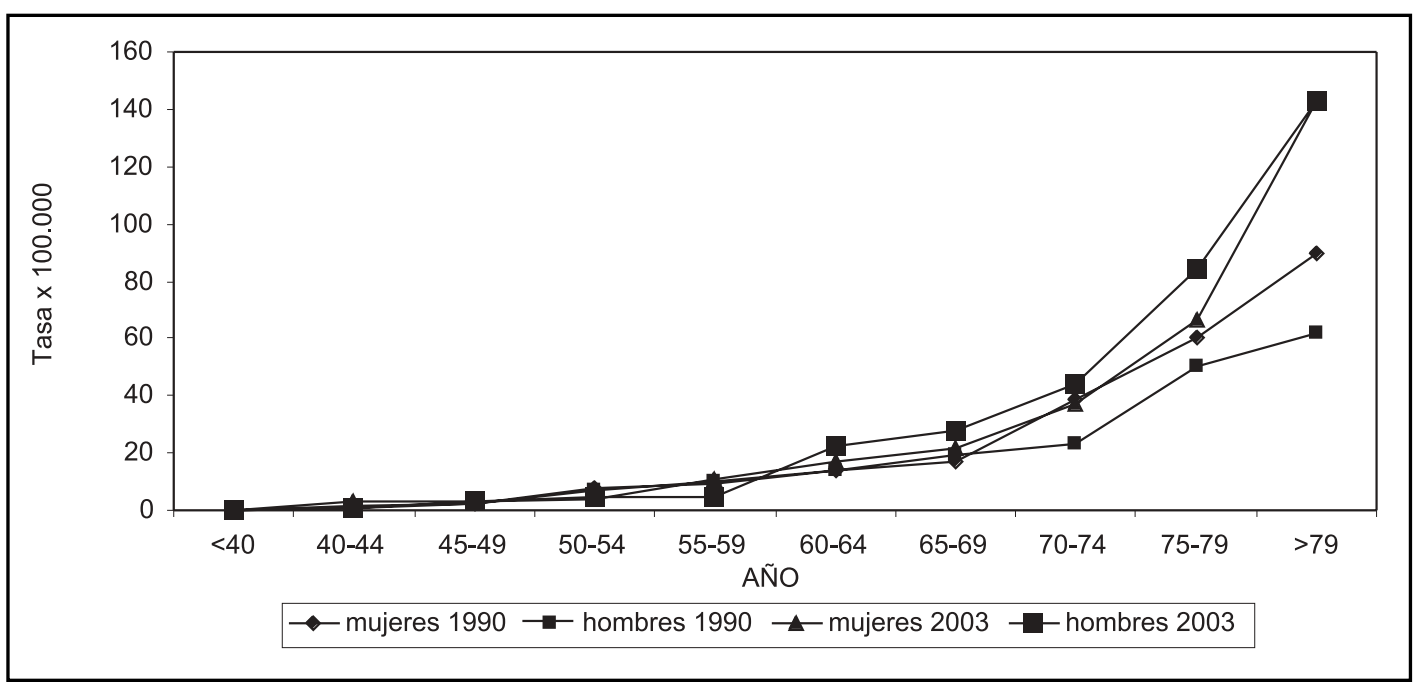

Figura 4. Tasas de mortalidad por cáncer de colon según sexo y rangos de edad. Chile 1990 y 2003. 


\section{DisCUSIÓN}

Este estudio demostró que en Chile, en el período 1990-2003, la mortalidad por cáncer de colon presentó una tendencia ascendente y significativa.

En ese período, el envejecimiento poblacional, considerando el aumento de la población de 50 o más años, la de mayor riesgo para cáncer de colon, aumentó de 2.110 .516 (16,0\% de la población) en 1990, a 3.152.245 (20,0\% de la población) en $2003^{9}$. Al ajustar las tasas por edad se observó una disminución de la pendiente, pese a eso, la tendencia persiste ascendente y significativa; esto significa que si bien el envejecimiento poblacional influye en el aumento de la mortalidad por cáncer de colon, existirían otros factores involucrados, como los cambios en los hábitos alimentarios registrados en la población chilena y que se asocian a esta patología ${ }^{12}$, y posiblemente por la falta de un programa específico de pesquisa oncológica.

En Chile no hay un registro nacional de cáncer, por lo que no se puede establecer la prevalencia de la enfermedad. Indirectamente, por los resultados de este estudio se podría inferir que la prevalencia también ha aumentado.

Como resultado secundario observamos que la mortalidad general por cáncer de colon en mujeres, fue significativamente mayor en cada año del período estudiado y no disponemos de argumentos definitivos que expliquen este hallazgo y lo presentamos como un fenómeno observacional a considerar en futuras investigaciones prospectivas. Además, pudimos comprobar que en la población mayor de 50 años se concentra más de $90 \%$ de la mortalidad por cáncer de colon, lo que justifica la pesquisa de esta patología a partir de esa edad.

En 2003, hubo 976 muertes por cáncer de colon, con un promedio de 2,7 muertes diarias, que, junto al aumento significativo de la mortalidad en el período estudiado, hace que estimemos que esta neoplasia debería ser considerada como un problema emergente de salud pública y debiera tener un programa específico para su control, como acontece con el cáncer cervicouterino y el de mama ${ }^{13}$. No debemos olvidar que este cáncer tiene largos períodos de fase asintomática, lesiones premalignas tratables y grupos identificables de alto riesgo ${ }^{14}$, cumpliendo por lo tanto con las características para establecer un programa de screening poblacional.

En países desarrollados, la pesquisa de cáncer de colon es controversial en lo relacionado a costo-efectividad y la técnica a usar. La detección de sangre oculta en deposiciones (prueba del guayaco) es la única metodología de screening con estudios randomizados que ha demostrado la reducción poblacional de las muertes por cáncer colorrectal ${ }^{6,7}$. Con esa técnica, un estudio efectuado en el Reino Unido en 271.646 personas de 50 a 69 años, permitió detectar 552 cánceres, el 48\% estaba en etapa A de Dukes y sólo 1\% presentaba metástasis al momento del diagnóstico ${ }^{15}$; el costo estimado del screening fue de US\$10.800 por año de vida salvado. En Hungría y EE.UU, estudios basados en modelos matemáticos, con la misma técnica, el costo estimado fue de US\$4.381 y US\$81.678 por año de vida salvado, respectivamente ${ }^{16,17}$. Un estudio de revisión sistemática efectuado por la US Preventive Services Task Force, concluyó que el screening para cáncer colorrectal era costo-efectivo en años de vida salvados comparado con no hacerlo ${ }^{18}$.

La Sociedad Americana del Cáncer recomienda como screening para población asintomática, mayor de 50 años, con riesgo estándar de cáncer colorrectal, las alternativas que se presentan en la Tabla $3^{19}$. Sin embargo, para hacer efectivas esas recomendaciones en Chile, hay que vencer una serie de barreras logísticas, como los problemas culturales de la población en relación a los métodos invasivos, disponer de suficiente infraestructura profesional para satisfacer la demanda de procedimientos endoscópicos, como también el costo para los servicios públicos y la cobertura de los seguros privados de salud.

El médico debe informar al paciente mayor de 50 años de los beneficios de la detección precoz de esta neoplasia, como también del costo, la seguridad y las molestias de los procedimientos. La información directa por el médico al paciente ha mostrado ser un factor determinante en la adherencia a los métodos de screening ${ }^{20,21}$. Mientras, se debe educar a la población en el control de hábitos y conductas que se relacionan con cáncer colorrectal, como son el hábito tabáqui$\mathrm{co}^{22}$, el consumo excesivo de carnes rojas y grasas $^{23}$, la obesidad ${ }^{24}$ y el sedentarismo ${ }^{25}$. 
Tabla 3. Recomendaciones de la Sociedad Americana del C áncer para la detección precoz del adenoma y cáncer colorrectal en población con riesgo promedio y mayores de 50 años

\begin{tabular}{|c|c|c|}
\hline Procedimiento & Intervalo & Comentario \\
\hline $\begin{array}{l}\text { Sangre oculta en } \\
\text { deposiciones más } \\
\text { sigmoidoscopia flexible }\end{array}$ & $\begin{array}{l}\text { - Sangre oculta en } \\
\text { deposiciones: anual } \\
\text { - Sigmoidoscopia: cada } \\
5 \text { años }\end{array}$ & $\begin{array}{l}\text { - Se prefiere la } \\
\text { combinación sobre las } \\
\text { pruebas solas. } \\
\text { - Colonoscopia en todas } \\
\text { las pruebas positivas. }\end{array}$ \\
\hline Sigmoidoscopia flexible & Cada 5 años & $\begin{array}{l}\text { - Colonoscopia en la } \\
\text { prueba positiva. }\end{array}$ \\
\hline $\begin{array}{l}\text { Sangre oculta en } \\
\text { deposiciones }\end{array}$ & Anual & $\begin{array}{l}\text { - } \quad \text { Se recomienda la toma } \\
\text { múltiple domiciliaria. } \\
\text { - } \\
\text { Colonoscopia en la } \\
\text { prueba positiva. }\end{array}$ \\
\hline Colonoscopia & Cada 10 años & $\begin{array}{l}\text { Permite visualizar, } \\
\text { biopsiar y/o remover las } \\
\text { lesiones significativas. }\end{array}$ \\
\hline $\begin{array}{l}\text { Enema baritado de doble } \\
\text { contraste }\end{array}$ & Cada 5 años & $\begin{array}{l}\text { - Colonoscopia en la } \\
\text { prueba positiva. }\end{array}$ \\
\hline
\end{tabular}

Fuente: American Cancer Society Guidelines ${ }^{19}$.

En conclusión, los resultados de este estudio permiten establecer que la mortalidad por cáncer de colon es un problema emergente de salud pública en Chile. Sugerimos que las instituciones

\section{REFERENCIAS}

1. Medina E, KaempFFer A. Mortalidad por cáncer en Chile: consideraciones epidemiológicas. Rev Méd Chile 2001; 129: 1195-202.

2. Cancermondial. Statistical Information System. International Agency for Research on Cancer. Disponible en: http://www-dep.iarc.fr/. Acceso: 10 de junio de 2005.

3. Jemal A, Timari RC, Murray T, Ghafoor A, Samuers A, WaRD E et al. Cancer Statistics, 2004. CA Cancer J Clin 2004; 54: 8-29.

4. Gibbons L, Mao Y, Eumson L. Trends in colorectal cancer incidence and mortality. Health Rep 2001; 12: 41-55. de salud deberían implementar a futuro un programa de pesquisa para cáncer de colon, única estrategia con evidencia que permite reducir las muertes por esta patología. 
ries and Causes of Death. Vol 1. 10 $0^{\text {th }}$ revision. Geneva, 1992.

11. Wei EK, Giovannucci E, Wu K, Rosner B, Fush CS, WILETT WC ET AL. Comparison of risk factors for colon and rectal cancer. Int J Cancer 2004; 108: 433-42.

12. Albala C, Vio F, Kain J, Uauy R. Nutrition transition in Chile: determinants and consequences. Public Health Nutr 2002; 5: 123-8.

13. Ministerio de Salud de Chile. Programa Nacional de Cáncer. Disponible en: www.minsal.cl. Acceso: 5 de enero de 2005.

14. MAYER RJ. Gastrointestinal tract cancer. En: Braunwald E, Fauci AS, Kasper DL, Hauser SL, Longo DL, Jameson JL (eds). Principles of Internal Medicine, Harrison's $15^{\text {th }}$ edition, Volume 1. The McGraw-Hill Companies, Inc., 2001; 578-88.

15. UK Colorectal Cancer Screening Pilot Group. Results of the first round of a demonstration pilot of screening for colorectal cancer in the United Kingdom. BMJ 2004; 329 (7458): 133. doi:10.1136/bmj.38153.491887.7C.

16. Boncz I, Sebestyén A, Dózsa CA, Pál M, Sándor J, PALÁsti J ET AL. Health economics analysis of colorectal screening. Hungarian Oncology 2004; 111-15.

17. SonNenBerg A, Delcò F, Inadomi JM. Cost-effectiveness of colonoscopy in screening for colorectal cancer. Ann Inter Med 2000; 133: 573-84.
18. Pignone M, Saha S, Hoerger T, Mandeiblatt J. Costeffectiveness analyses of colorectal cancer screening: a systematic review for the US. Preventive Services Task Force. Ann Inter Med 2002; 137: 96-104.

19. Smith RA, Von Eschenbach AC, Wender R, Levin B, Byers T, Rothenberger T et al. American Cancer Society Guidelines for early detection of cancer: Update of early detection guidelines for prostate, colorectal and endometrial cancers. CA Cancer J Clin 2001; 51: 38-75.

20. VERNON SW. Participation in colorectal cancer screening: a review. J Nat Cancer Inst 1997; 89: 1406-22.

21. Pignone M, Bucholtz D, HarRis R. Patient preferences for colon cancer screening. J Gen Intern Med 1999; 14: 432-7.

22. Colangelo LA, Gapstur SM, Gann PH, Dyer AR. Cigarette smoking and colorectal carcinoma with long-term follow-up. Cancer 2004; 100: 288-93.

23. Bruce WR, Giacca A, Medune A. Possible mechanisms relating diet and risk of colon cancer. Cancer Epidemiol Biomark Prev 2000; 9: 1271-9.

24. Calue EE, Rodríguez C, Walker-Thurmond K, Thun MJ. Overweight, obesity, and mortality from cancer in a prospectively studied cohort of US adults. N Engl J Med 2003; 348: 1625-38.

25. BAUMAN AE. Updating the evidence that physical activity is good for health: an epidemiological review 2000-2003. J Sci Med Sport 2004; 7(1 Suppl): 6-19. 